abnormalities have optimal seizure control following surgical resection of the localized tuber. The localization of TSC lesions not showing concordant findings on MRI and EEG requires presurgical testing by surface video-EEG, PET, ictal SPECT, diffusion-weighted MRI, or intraoperative ECoG (Romanelli P et al. Pediatr Neurol 2004;31:239-247; Ped Neur Briefs 2004;18:77-78). In this previous study, resection of tubers and epileptogenic foci localized by the above methods resulted in control of seizures in $78 \%$ and reduction of seizure frequency in $20 \%$. Early detection and resection of refractory seizure foci can prevent deterioration of cognitive functioning, improve behavior, and lead to a better quality of life.

\title{
NEUROPATHIES
}

\section{SUBACUTE DEMYELINATING POLYNEUROPATHY}

The electroclinical characteristics of 5 children (ages 4-13 years) with subacute inflammatory demyelinating polyneuropathy (SIDP) are reported from the Royal Children's Hospital, Victoria, Australia. Onset followed 2-13 days after a nonspecific infection with fever, upper respiratory tract symptoms or gastroenteritis in 3 patients; one had recent cytomegalovirus infection; none had Campylobacter jejuni. All had leg weakness (with pain in 3) at presentation, and upper limb involvement followed. All remained ambulant. Neurologic findings included areflexia in 5, ataxia in 4, and bilateral facial weakness in 1 . CSF protein was elevated $(0.75-1.5 \mathrm{~g} / \mathrm{L})$ in 3 , and leukocytes were absent or $1 / \mathrm{mm}^{3}$. Nerve conduction studies were abnormal. Period from onset of symptoms to treatment was 4-8 weeks. All received oral prednisolone for 1.5-6 months, and all showed improved muscle strength within 1 week. Three had returned to normal within 6 months and one within 8 months, and none showed relapse at 6-20 years follow-up. One was normal when last seen at 10 months follow-up. (Rodriguez-Casero MV, Shield LK, Kornberg AJ. Subacute inflammatory demyelinating polyneuropathy in children. Neurology May (2 of 2) 2005;64:1786-1788). (Dr AJ Kornberg, Department of Neurology, Royal Children's Hospital, Flemington Rd, Parkville, Victoria, Australia 3052).

COMMENT. The authors have differentiated this series of SIDP patients with a benign, subacute illness and monophasic course of 4-8 weeks from the acute form of inflammatory demyelinating polyneuropathy (Guillain-Barre syndrome [GBS]) witì à 4 week course, and a chronic form (CIDP) with progression over 8 weeks and frequent relapse. SIDP characteristics that differ from GBS include 1) longer period of progression; 2) lack of respiratory, cranial nerve, or autonomic involvement; 3 ) more abnormal nerve conduction in initial stages; and 4) rapid and sustained response to corticosteroids. The monophasic and nonrelapsing course of SIDP differentiates it from CIDP. The response of CIDP to steroids is reviewed in Ped Neur Briefs 2005;19:19).

\section{EARLY ONSET CHARCOT-MARIE-TOOTH DISEASE}

The clinical signs and genetic analysis of early-onset Charcot-Marie-Tooth disease (CMT) in a 2-year-old boy and members of his family are reported from the Academic Medical Center, Amsterdam, and Sophia Children's Hospital, Rotterdam, the Netherlands. 